intensive care unit, drainage of the pleural effusion was performed with the application of alteplase for three days, continued treatment with antibiotics and symptomatics after confirmation of isolation of S. Pneumoniae from blood culture. On the tenth day of treatment, the boy became febrile with clinical deterioration. Radiologically, cavitation of the lung parenchyma was found, $4 \times 5 \mathrm{~cm}$ with an aeroliquid level, which corresponded to a lung abscess. Treatment continued with modification of antibiotic therapy, vancomycin was introduced. Parenteral antimicrobial treatment lasted for about 5 weeks and then continued orally (amoxicillin + clavulanic acid) for a total of 8 weeks. During the treatment, regression of pulmonary changes was monitored using ultrasound, and a control lung scan was taken after two months confirming the abscess regression.

Pulmonary abscess as a complication of pneumonia is not common but should be considered in any severe clinical presentation of inflammation accompanied by the development of complications, especially in children who have not been vaccinated against pneumococcus as was the case with our patient.

\section{SODIUM DEPLETION IN CYSTIC FIBROSIS PATIENTS OLDER THAN 24 MONTHS}

Ivan Bambir*, Bambir Ivan, Omerza Lana, Sapina Matej, Vukic Dugac Andrea, Tjesic Drinkovic Dorian, Tjesic - Drinkovic Duska. University Hospital Centre Zagreb

\subsection{6/archdischild-2021-europaediatrics.414}

Sodium depletion is a well-recognized complication of cystic fibrosis in infants and toddlers, due to which they are routinely prescribed sodium supplementation. It exists in normonatremic subjects as well. Data on sodium homeostasis in older age groups is scarce. Spot urine sodium/creatinine ratio $(\mathrm{UNa} / \mathrm{Cr})$ strongly correlates with the fractional excretion of sodium

(FENa) and is considered to be a marker of normonatremic sodium depletion $(\mathrm{NNaD}) .{ }^{1}$ This is a pilot observational study investigating the $(\mathrm{UNa} / \mathrm{Cr})$ in subjects older than 24 months, as a possible sign of sodium depletion.

Participants were children and adolescents seen between January 1st and October 31st 2019. at the UHC Zagreb - CF centre, Dept. of paediatrics.

Basic demographic, anthropometric clinical and laboratory data were collected. $\mathrm{UNa} / \mathrm{Cr}$ ratio was considered pathological if $<17 \mathrm{mmol} / \mathrm{mmol}$, corresponding to minimal target $\mathrm{FENa}$ of $0.5 \%$. Descriptive statistics and Mann Whitney U test were used for data analysis; the significance set at $\mathrm{p}<0.05$.

30 patients ( 13 male) aged from 2.25 to 21.5 years entered the study. Only one patient had $\mathrm{UNa}<10 \mathrm{mmol} / \mathrm{l}$. The calculated $\mathrm{UNa} / \mathrm{Cr}$ ratio ranged from 1.85 - $52.8 \mathrm{mmol} / \mathrm{mmol}$. $\mathrm{UNa} / \mathrm{Cr}$ below the lower limit of $17 \mathrm{mmol} / \mathrm{mmol}$ was found in 20/30 patients (median $6.41 \mathrm{mmol} / \mathrm{mmol}$; IQR 4.24 7.39). One third of subjects (10/30) had $\mathrm{UNa} / \mathrm{Cr}$ above the cut-off value with the median value $26.04 \mathrm{mmol} / \mathrm{mmol}$ (IQR 23.55 - 50.54). We observed an almost significant difference in the age of patients with and without decreased $\mathrm{UNa} / \mathrm{Cr}$ : median age in decreased $\mathrm{UNa} / \mathrm{Cr}$ ratio group was 14.65 years (IQR 8.24 - 19.76) vs. 8.675 years (IQR 6.1 - 12.5) in the normal reference group $(\mathrm{p}=0,0556)$.

Plasma sodium concentration was measured in 20/30 patients and all samples were within the normal reference range $(135 \mathrm{mmol} / \mathrm{l}$ to $144 \mathrm{mmol} / \mathrm{l})$, regardless whether the
$\mathrm{UNa} / \mathrm{Cr}$ was normal $(\mathrm{n}=7)$, or below $17 \mathrm{mmol} / \mathrm{mmol}$ $(n=13)$.

Measurement of $\mathrm{UNa}$ alone is a rough parameter of sodium balance; in our set of patients, this criterion identified only $1 / 30$ subjects as sodium depleted. However, we found a significant proportion of tested subjects (20/30) with an abnormally low $\mathrm{UNa} / \mathrm{Cr}$, suggesting an abnormally low fractional excretion of sodium - a parameter that reflects sodium status more appropriately. The plasma sodium concentrations remained normal. We suggest that this is due to an increase in sodium reabsorption to control hyponatremia. $\mathrm{NNaD}$ may be an underrecognized condition among CF patients beyond toddlers' age.

\section{REFERENCE}

1. Coates, et al. J Cys Fib 2009;382-5.

\section{PRIMARY CILIARY DYSKINESIA - A THERAPEUTIC CHALLENGE}

Ivan Bambir*, Jadranka Kelecic, Sanda Huljev Frkovic, Dorian Tjesic-Drinkovic. University Hospital Centre Zagreb

10.1136/archdischild-2021-europaediatrics.415

Primary ciliary dyskinesia (PCD) is a hereditary heterogeneous pulmonary disease characterized by abnormalities of ciliary biogenesis, ultrastructure and function, leading to the development of bronchiectasis and chronic sinusitis, with situs inversus in about half of the patients. Diagnosis is based on a combination of phenotypic characteristics and diagnostic tests.

Treatment is directed to preserve pulmonary function and delay chronic changes leading eventually to lung transplantation. Therapeutic recommendations are based on the experience regarding treating diseases with similar mucociliary clearance disorders.

Here we describe the treatment course of a girl with PCD with situs viscerum inversus, whose diagnosis was confirmed at the age of 6 months by biopsy of the airway mucosa and electron microscopy showing lack of inner dynein arms.

The patient had respiratory difficulties since birth and chronic cough. The chest X-ray repeatedly showed pulmonary paracardial shading of the left lung. Ad-hoc physical therapy, secretolics, inhaled steroids, salbutamol and, if necessary, antibiotics were prescribed. At 7 years of age, MSCT of the thorax revealed advanced left-sided pathology: parenchymal consolidation of the middle lobe with air bronchogram and ectatic bronchi and in the lower lobe ectatic bronchi and small-nodular interstitial infiltrates.

According to these findings, therapeutic measures were intensified with daily physiotherapy including high-frequency thoracic drainage (Vest machine), Flutter, manual drainage and encouraging physical activity. The use of secretolytics with antibiotics (orally, i.v., inhalations) according to sputum microbiology was regular and macrolide as immunomodulatory therapy was introduced. Checkups with spirometry and sputum analysis were performed every 3 months and ENT controls 12 times yearly. She was vaccinated against pneumococcus and yearly against influenza.

After 3.5 years of this regular treatment and monitoring, a new CT-scan showed significant resolution of the consolidation in middle pulmonary lobe with now visible bronchiectasis and wall thickening, as well as complete resolution of parenchymal lesions in the posterobasal segment of the left inferior lobe. In 